\title{
Metabolomic Analysis of Complex Chinese Remedies: Examples of Induced Nephrotoxicity in the Mouse from a Series of Remedies Containing Aristolochic Acid
}

\author{
Dong-Ming Tsai, ${ }^{1,2}$ Jaw-Jou Kang, ${ }^{3}$ Shoei-Sheng Lee, ${ }^{4}$ San-Yuan Wang, \\ I-Lin Tsai, ${ }^{2,4}$ Guan-Yuan Chen, ${ }^{2,4}$ Hsiao-Wei Liao, ${ }^{4}$ Li Wei-Chu, ${ }^{6}$ \\ Ching-Hua Kuo, ${ }^{2,4}$ and Y. Jane Tseng ${ }^{1,2,4,5}$ \\ ${ }^{1}$ Graduate Institute of Biomedical Electronics and Bioinformatics, National Taiwan University, No. 1, Sec. 4, \\ Roosevelt Road, Taipei 106, Taiwan \\ ${ }^{2}$ The Metabolomics Core Laboratory, Center of Genomic Medicine, National Taiwan University, No. 1, Sec. 4, \\ Roosevelt Road, Taipei 106, Taiwan \\ ${ }^{3}$ Institute of Toxicology, College of Medicine, National Taiwan University, No. 1, Sec. 4, \\ Roosevelt Road, Taipei 106, Taiwan \\ ${ }^{4}$ Department of Pharmacy, College of Medicine, National Taiwan University, No. 1, Sec. 4, Roosevelt Road, Taipei 106, Taiwan \\ ${ }^{5}$ Department of Computer Science and Information Engineering, National Taiwan University, No. 1, Sec. 4, \\ Roosevelt Road, Taipei 106, Taiwan \\ ${ }^{6}$ Sheng Chang Pharmaceutical Co., Ltd., Jung-Li, Taiwan
}

Correspondence should be addressed to Y. Jane Tseng; yjtseng@csie.ntu.edu.tw

Received 8 January 2013; Revised 26 February 2013; Accepted 27 February 2013

Academic Editor: Mark Moss

Copyright (C) 2013 Dong-Ming Tsai et al. This is an open access article distributed under the Creative Commons Attribution License, which permits unrestricted use, distribution, and reproduction in any medium, provided the original work is properly cited.

\begin{abstract}
Aristolochic acid nephropathy is caused by aristolochic acid (AA) and AA-containing herbs. In traditional Chinese medicine, a principle called "Jun-Chen-Zou-Shi" may be utilized to construct a remedial herbal formula that attempts to mitigate the toxicity of the main ingredient. This study used Bu-Fei-A-Jiao-Tang (BFAJT) to test if the compound remedy based on a principle of "JunChen-Zou-Shi" can decrease the toxicity of AA-containing herbs. We compared the three toxicities of AA standard, Madouling (an Aristolochia herb), and a herbal formula BFAJT. AA standard was given for BALB/c mice at a dose of $5 \mathrm{mg} / \mathrm{kg} \mathrm{bw} / \mathrm{day}$ or $7.5 \mathrm{mg} / \mathrm{kg}$ bw/day for 10 days. Madouling and BFAJT were given at an equivalence of AA $0.5 \mathrm{mg} / \mathrm{kg}$ bw/day for $21 \mathrm{days}$. Nephrotoxicity was evaluated by metabolomics and histopathology. The urinary metabolomics profiles were characterized by ${ }^{1} \mathrm{H}$ NMR spectroscopy. The spectral data was analyzed with partial least squares discriminant analysis, and the significant differential metabolites between groups were identified. The result showed different degrees of acute renal tubular injuries, and metabolomics analysis found that the kidney injuries were focused in proximal renal tubules. Both metabolomics and pathological studies revealed that AA standard, Madouling, and BFAJT were all nephrotoxicants. The compositions of the compound remedy did not diminish the nephrotoxicity caused by AA.
\end{abstract}

\section{Introduction}

Aristolochic acids (AAs) are potent nephrotoxic agents [1,2] that are found primarily in the plant genera Aristolochia and Asarum $[3,4]$. These herbs have been used as a component of herbal remedies in traditional Chinese medicine (TCM). Herbal remedies containing Aristolochia and Asarum have been used to relieve symptoms such as cough, arthritic pain, and gastrointestinal problems $[5,6]$. However, chronic kidney injury may occur in humans after a prolonged intake of these Aristolochiaceous herbs. Inadvertent replacement of Stephania tetrandra by Arsitolochia fangchi has caused rapidly progressive interstitial renal fibrosis (also named Chinese herbs nephropathy) in young women on a slimming regimen 
$[7,8]$. AAs were determined to be the major components that caused the toxicity [9]. The kidney injury induced by AAcontaining herbs characterized by tubulointerstitial injury and paucity of filtration of inflammatory cells in the kidney is named aristolochic acid nephropathy (AAN) [10,11]. Until prohibited, these herbs were widely used worldwide and victims of AAN had been reported in many countries $[2,8$, 12].

TCMs are generally used as compound remedies which are composed of several herbals. Chinese herbal classics indicate that the components in herbal remedies can be divided into the 4 principles: "Jun-Chen-Zou-Shi" which represents "the emperor, the minister, the assistant and the courier" $[13,14]$. The emperor herbs (Jun) are the main components to relief symptoms. The minister herbs (Chen) act as an adjunct to facilitate the emperor herbs in relief of symptoms. The assistant herbs (Zou) help to enhance the efficacy provided by Jun and Chen and to counteract toxic and side effects caused by these herbs. The courier herbs (Shi) act as an emollient for the herbal remedy [15]. AAcontaining herbs are generally used as compound remedies. Many studies have been performed on the nephrotoxicity of pure aristolochic acid, but there is very limited nephrotoxicity information regarding the commonly used medicinal herbs of Aristolochiaceae or the compound remedies containing the Aristolochiaceae herb. Before AA was outlawed, several herbal formulas containing AA had been used in TCM. Longdan Xieganwan is a TCM formula, which contains Caulis Aristolochiae manshuriensis among its 10 ingredients. The remedy was used as a "liver enhancer" and its toxicity was supposed to be lessened via the combination of other components according to the Jun-Chen-Zou-Shi theory. However, Londan Xieganwan had been reported to be toxic in humans and rats [16-18]. These observations made the concept of using compound remedies to reduce AAN a debatable issue. Another herbal formula Bu-Fei-A-Jiao-Tang (BFAJT) which is a decoction containing Fructus Aristolochia contorta (Madouling) is used for some lung-related symptoms [19]. No study discusses the toxicity of this herbal formula until now.

Metabolomics is a newly developed technology to study phenotype changes of the cellular responses to pathophysiological stimuli or genetic modification through a holistic metabolite analysis $[20,21]$. The metabolite profile comprises hundreds to thousands of endogenous organic metabolites. Through analytical platforms such as proton nuclear magnetic resonance $\left({ }^{1} \mathrm{H}-\mathrm{NMR}\right)$ or hyphenated liquid chromatography with mass spectrometry (LC-MS), metabolite profiles can be obtained $[22,23]$. With the advance of computers and chemometric techniques, the complex data resulting from these platforms can be mined for useful information [24].

Several studies have applied a metabolomics approach to study AA toxicity. Zhang et al. reported that AA given rats showed significant renal toxicity with a metabolite pattern similar to other proximal renal tubular toxicants through ${ }^{1} \mathrm{H}$ NMR spectroscopic metabolomic study [25]. Liang et al. used ${ }^{1} \mathrm{H}$ NMR to study renal toxicity of Aristolochia fangchi in rats, and the AA equivalent dose they used was
$3.7 \mathrm{mg} / \mathrm{kg} /$ day for 4 weeks. Renal toxicity was detected at 2 weeks in their study [26]. Chen et al. used LC-MS to investigate AA and Aristolochia manshuriensis nephrotoxicity in rats, and the equivalent AA dose of $A$. manshuriensis they used was $96 \mathrm{mg} / \mathrm{kg} /$ day for 4 consecutive days. They indicated that a metabolomics approach is promising in providing rapid screening of nephrotoxicity [27].

To test if the compound remedy based on a principle of "Jun-Chen-Zou-Shi" can decrease the toxicity of AA containing herbs, we used BFAJT as our test remedy to study its nephrotoxicity by metabolomics. The advantages of using ${ }^{1} \mathrm{H}$-NMR experiment in metabolomics include simple sample preparation and high system robustness [28]. It detects the resonance signal of different proton groups and can provide the structural information of metabolites. ${ }^{1} \mathrm{H}-\mathrm{NMR}$ was applied to obtain the urinary metabolic profiles of mice treated with herbs. This study anticipates providing scientific evidence of nephrotoxicity of BFAJT.

\section{Materials and Methods}

2.1. Animal Handling and Sampling. Animal care and handling protocols were in compliance with national animal treatment guidelines and approved by the Animal Committee of National Taiwan University. All animal studies were performed in the animal center of National Taiwan University Medical College Animal Center. A total of 24 male BALB/c mice aged 6-8 wk (18-20 g) were obtained from the Laboratory Animal Center, Medical College of National Taiwan University, Taipei, Taiwan. Regular rodent laboratory chow (Purina Mills, Inc., St. Louis, MO) and water were allowed freely. Animals were lodged in individual metabolic cage and acclimated in temperature $25^{\circ} \mathrm{C}$ and humidity $60 \%$ with regular day/dark light cycle, starting from one week before each experiment to reduce the stress of adjusting to new environment for animals. Same conditions were used throughout the experiments.

2.2. Chemicals and Herbal Materials. Authentic pure reference aristolochic acid, Madouling (Fructus Aristolochia contorta), and a compound remedy Bu-Fei-A-Jiao-Tang (BFAJT) were used in this study. Aristolochic acid was purchased from Acros Organics (NJ, USA). The content is AA-I 96\% (90.9\%) and AA-II 4\% (5.7\%). AA-I is the major constituent of AAs in our test standard and it is also the major aristolochic acid component in the tested herb. Therefore, an AA-I equivalent dose was used to control the AA administration dose for mice fed with AA standard, Madouling, and BFAJT. Madouling powder was purchased from Sheng Chang Pharmaceutical Co., Ltd. (Chung-Li, Taiwan). The dried decoction powder was filtered and extracted from boiled herb. The dosing sample was a mixture of the decoction and corn oil. The content of AA-I is $24.17 \mathrm{mg} / \mathrm{gm}$ and of AA-II is $2.04 \mathrm{mg} / \mathrm{g}$ for the dosing sample. BFAJT powder was purchased from Sheng Chang Pharmaceutical Co. The dried decoction powder was processed using the same procedure as that of Madouling. The content of AA-I is $3.749 \mathrm{mg} / \mathrm{g}$ and of AA-II is $0.169 \mathrm{mg} / \mathrm{g}$ for the dosing sample. The BFAJT powder is composed of 
donkey hide gelatin $45 \mathrm{~g}$, Madouling $15 \mathrm{~g}$, apricot seed $6 \mathrm{~g}$, great burdock fruit $7.5 \mathrm{~g}$, rice $30 \mathrm{~g}$, and honey fried licorice root $7.5 \mathrm{~g}$.

2.3. HPLC Conditions. The equipment consisted of a pair of ShimadzuLC-10 AT pumps (Kyoto, Japan), a Rheodyne 7725i5-mL manual injector (Cotati, CA, USA), and a Shimadzu SPD-M10A diode array detector. Separations were carried out on a Luna C column, $250 * 4.6 \mathrm{~mm}, 5 \mu \mathrm{m}$ (Phenomenex, Torrance, CA, USA). The mobile phase was composed of $0.7 \%$ acetic acid and acetonitrile, $57: 43(\mathrm{v} / \mathrm{v})$.

2.4. Experiment Design. The experiments were divided into two parts. Experiment 1 investigated the toxicity of AA reference standard. Nine mice were randomly divided into 3 groups. They were control group $(n=3)$ treated with vehicle of corn oil, the middle dosed group $(n=3)$ treated with AA $5 \mathrm{mg} / \mathrm{kg}$ bw per day, and the high dosed AA group $(n=3)$ treated with AA $7.5 \mathrm{mg} / \mathrm{kg}$ bw per day. The three groups were tagged as AA0, AA5, and AA7.5. AA was dissolved in corn oil with a concentration of 1 and $1.5 \mathrm{mg} / \mathrm{mL}$. The vehicle and AA were given to mouse via oral gavage once daily. Urine samples were collected on days 1, 3, 8, and 10 after dosing. The collected urine was centrifuged at $3000 \mathrm{rpm}$ for $15 \mathrm{~min}$ immediately, and the clear suspension was stored at $-80^{\circ} \mathrm{C}$ after adding sodium azide to reach a final concentration of $10 \mathrm{mM}$ of sodium azide. All mice were euthanized after experiment for renal histopathological analysis at 10 days after dosing. Urine samples were sent for NMR analysis. Body weights were measured on selected days.

Experiment 2 investigated toxicity of AA containing herbals in low AA dosage. The equivalence dose of AA for both Madouling group and BFAJT group is $0.5 \mathrm{mg} / \mathrm{kg}$ bw per day. Nine mice were randomly divided into three groups; they were control group ( $n=3)$, treated with vehicle, Madouling dosed group $(n=3)$, treated with Madouling powder $400 \mathrm{mg} / \mathrm{kg}$ bw per day, and BFAJT dosed group $(n=$ 3 ), treated with BFAJT $4 \mathrm{~g} / \mathrm{kg}$ bw per day. The 3 groups were tagged as $\mathrm{C} 0, \mathrm{M} 0.5$, and $\mathrm{BF} 0.5$. The equivalent amount of AA for Madouling $400 \mathrm{mg}$ and BFAJT $4 \mathrm{~g}$ is $0.5 \mathrm{mg}$. All substances were dissolved in corn oil and given through oral gavage once daily. Collection and handling of urine was similar as described in the first part experiment. Urine samples collected at days $1,3,10$, and 13 were sent for ${ }^{1} \mathrm{H}$ NMR spectroscopy. All mice were euthanized at day 20 after dosing, and a histopathological study of the kidneys was performed. A summary of these two experiments is described in Table 1.

2.5. Renal Histopathology. The section of formalin-fixed paraffin-embedded kidney tissue was stained with hematoxylin/eosin. The stained kidney sections were analyzed under a light microscope. The degree of renal lesions was graded from one to five depending on the severity: $1=$ minimal (<1\%); 2 : slight $(1 \%-25 \%) ; 3=$ moderate $(26 \%-50 \%)$; $4=$ moderate/severe $(51 \%-75 \%) ; 5=$ severe/high $(76 \%-100 \%)$ [29]. It was according to the renal histopathological findings of anatomical site of lesion (cortex to medulla), location of renal tubular lesion (proximal to distal, focal to locally
TABLE 1: Experiment design.

\begin{tabular}{|c|c|c|c|}
\hline Group $^{a}$ & Substance & $\begin{array}{l}\text { Eq. dose to AA } \\
\text { (mg/kg bw/day) }\end{array}$ & Urine sampling date \\
\hline \multicolumn{4}{|c|}{ Experiment 1} \\
\hline AA0 & Vehicle & 0 & Day $1,3,8,10^{\mathrm{b}}$ \\
\hline AA5 & $\mathrm{AA}$ & 5.0 & Day $1,3,8,10^{\mathrm{b}}$ \\
\hline AA7.5 & AA & 7.5 & Day $1,3,8,10^{\mathrm{b}}$ \\
\hline \multicolumn{4}{|c|}{ Experiment 2} \\
\hline C0 & Vehicle & 0 & Day $1,3,10,13^{c}$ \\
\hline M0.5 & Madouling & 0.5 & Day $1,3,10,13^{c}$ \\
\hline BF0.5 & BFAJT & 0.5 & Day $1,3,10,13^{c}$ \\
\hline
\end{tabular}

${ }^{\mathrm{a}}$ Three mice/group. ${ }^{\mathrm{b}}$ Mice of AA groups were euthanized on day 10 for renal histopathology.

${ }^{\mathrm{c}}$ Mice of all groups were euthanized on day 20.

AA: aristolochic acid; BFAJT: Bu-Fei-A-Jiao-Tang.

extensive), morphology of renal tubular lesion (dilatation with or without hyaline cast to necrosis), and patterns of inflammation (acute to subacute).

2.6. NMR Spectroscopic Analysis of Urine. ${ }^{1} \mathrm{H}$ NMR spectroscopy was performed from collected urine samples. A test sample of $825 \mu \mathrm{L}$ for each mouse was prepared using $500 \mu \mathrm{L}$ of the urine sample, $250 \mu \mathrm{L}$ of $0.2 \mathrm{M} \mathrm{Na}_{2} \mathrm{HPO}_{4}(\mathrm{pH} \mathrm{7.4)}$, and $75 \mu \mathrm{L}$ of sodium 3-trimethylsilyl-1-(2, 2, 3, 3- $\left.d_{4}\right)$ propionate (TSP) in $\mathrm{D}_{2} \mathrm{O}$ (final concentration $0.1 \mathrm{mg} / \mathrm{mL}$ ). $\mathrm{D}_{2} \mathrm{O}$ provided an NMR lock signal for the NMR spectrometer. Conventional ${ }^{1} \mathrm{H}$ NMR spectra of the urine samples were obtained from a Bruker Avance 600 spectrometer (Bruker Biospin, Germany) operated at $600.04 \mathrm{MHz}$ at $25^{\circ} \mathrm{C}$. One-dimensional ${ }^{1} \mathrm{H}$ NMR spectra were acquired using a standard NOESYPR1D pulse sequence (recycle delay- $90^{\circ}-t_{1}-90^{\circ}-t_{m}-90^{\circ}$-acquisition; XWIN-NMR3.5) with a recycle delay time of $2 \mathrm{~s}$, and a mixing time of $150 \mathrm{~ms}$. The $90^{\circ}$ pulse length was adjusted to $\sim 12.5 \mu \mathrm{s}$ at $-1 \mathrm{~dB}$ and $t_{1}$ was set to $3 \mu \mathrm{s}$, which provided an acquisition time of $2.72 \mathrm{~s}$. The FIDs were multiplied by an exponential weighting function corresponding to a line broadening of $0.3 \mathrm{~Hz}$, and the data were zero-filled to $64 \mathrm{k}$ data points. All spectra were corrected for phase and baseline distortions and referenced to the internal reference standard TSP $\left(\delta^{1} \mathrm{H}=0.0\right)$. Each ${ }^{1} \mathrm{H}$ NMR free induction decay (FID) data was transformed to $1 \mathrm{D}$ spectrum in ACD/Labs v10.0 1D NMR manager (Advanced Chemistry Development, Inc., Canada). The spectral data was exported to a $16 \mathrm{k}$ data points text file recording chemical shifts and their respective signal intensities. Baseline correction and binning were performed using an in-house script under the $R$ statistical environment (version 2.11.1) [30]. The spectral intensities were binned in $0.04 \mathrm{ppm}$ from $0 \mathrm{ppm}$ to $10 \mathrm{ppm}$ and scaled. Intensity data of water (4.5-5.5 ppm) and urea (5.5-6.0 ppm) were set to zero. To normalize metabolite concentration among these spectra, a probabilistic quotient normalization algorithm was performed [31].

2.7. Multivariate Analysis. Partial least squares discriminant analysis (PLS-DA) is a common approach to multivariate 
metabolomics data analysis. PLS analysis maximizes the product of variance matrix of measured variables (e.g., NMR metabolomic profile data) and correlation of measured data with properties of interest (e.g., toxicity), while DA predicts class membership of a dataset $\mathrm{X}$ with a y vector including only 0 and 1 ( 1 indicates that one sample belongs to a given class). For more than 2 classes, the PLS2 algorithm was applied [32]. PLS-DA was performed using the pls package (version 2.10) [33] in R. Validation of PLS-DA classification models was performed by cross model validation using the method of Westerhuis et al. [34]. In addition, a permutation test is applied with 2,000 random assignments of classes. The test set sample classification errors were evaluated to qualify the classification results. Scoring plots with two components were drawn for spectral classification. Loadings plots were drawn to search for significant chemical shift variables. To evaluate the fitness of the model, values of explained variation, $R^{2}>$ 0.7 , and predicted variation, $Q^{2}>0.4$ is considered as a good model [35].

Urine metabolites were assigned by referencing peak pattern and chemical shift from the NMR library of Human Metabolic Database (HMDB) [36], Chenomx NMR Suite Professional software package version 7.0 (Chenomx Inc., AB, Canada) and previous reports on rodent urine ${ }^{1} \mathrm{H}$ NMR in the literature $[37,38]$.

Paired univariate binned NMR data between groups was analyzed with a nonparametric Wilcoxon rank-sum test. Bins with $P$ value less than 0.05 were considered as significant metabolites.

\section{Results and Discussion}

The experimental design is shown in Table 1. Experiment 1 was designed as the positive control, and the two dosing groups of 0,5 , and $7.5 \mathrm{mg} / \mathrm{kg}$ bw/day of AA standard in mice. HPLC was used to quantify the content of AAs in Madouling and BFAJT. Experiment 2 was designed to evaluate the nephrotoxicity of AAs containing herbs. The contents of AA-I and AA-II in Madouling are 1.051 and $0.089 \mu \mathrm{g} \mathrm{mg}^{-1}$, respectively. The contents of AA-I and AAII in BFAJT are 0.113 and $0.012 \mu \mathrm{g} \mathrm{mg}^{-1}$, respectively. AA-I is the major constituent of AAs in our test standard, and it is also the major aristolochic acid component in the tested herb. Therefore, an AA-I equivalent dose was used to control the AA administration dose for mice fed with AA standard, Madouling and BFAJT. Considering the daily maximum feeding amount for mice, the AA-I equivalent doses for Madouling, and BFAJT groups were $0.5 \mathrm{mg} / \mathrm{kg}$ bw/day in Experiment 2.

3.1. Physiological Changes and Pathology. The body weight of mice treated with different doses of AA standard showed no significant changes from day 0 to day 9. Renal pathology revealed that the control group (treated with vehicle only) showed normal morphology on day 10. Mice treated with AA standard and herbals showed kidney injuries of different degrees. In Experiment 1, both AA standard treated groups (AA5 and AA7.5) showed a grade 3-4 severe shrinking of the proximal tubular cytoplasm and atrophy plus luminal dilation of the distal tubular system in large parts. Despite this acute renal tubulointerstitial injury, the glomerular morphology was relatively preserved in the AA dosed groups, and inflammatory cell infiltration was not prominent (Figures 1(a), 1(b), and 1(c)). This pathological change is similar to other AAN studies both on humans and mice [2,39].

In Experiment 2, after 20 days of vehicle treatment, normal renal pathohistology was observed for the control group. For mice treated with Madouling, the proximal renal tubules showed slight acute tubular degeneration and cellular swelling focally. In the BFAJT treated group, kidneys of mice showed similar mild proximal renal tubular injury as in the Madouling group. In all groups, no significant glomerular changes were observed (Figures 1(d), 1(e), and 1(f)). In summary, under treatment of high dose of AA standard (AA $5.0 \mathrm{mg} / \mathrm{kg}$ bw/day or higher), the kidney showed severe acute renal tubulointerstitial injuries. For mice treated with Madouling and BFAJT (AAI equivalent dose $0.5 \mathrm{mg} / \mathrm{kg}$ bw/day), the renal tubular lesions showed mild change at day 20 for both groups. In this study, the accumulative dosage of AA standard to induce acute renal histopathological changes was $50-75 \mathrm{mg} / \mathrm{kg}$ bw, which was equivalent to LD50 reported by Mengs given by a single oral dose [1]. Renal tubular atrophy and interstitial fibrosis were also observed by other studies with intraperitoneal injection of AA $[39,40]$. Shibutani et al. tested the mouse by orally administered $2.5 \mathrm{mg} / \mathrm{kg} /$ day of AAI and found severe renal tubular injuries with little interstitial inflammation at 10 days [41]. Compared to Experiment 1, the acute AA nephropathy was minor in Experiment 2 due to lower AA administration dose. The administration dose of AA in Experiment 2 was restricted by maximum feeding amount for mice, since the contents of AAI in Madouling and BFAJT were only 1.051 and $0.113 \mu \mathrm{g} \mathrm{mg}^{-1}$, respectively. Even though the administration dose was low, renal tubular lesions were still observed in kidneys.

3.2. Metabolic Changes in Urine Samples by ${ }^{1} H-N M R$. Mice urine was collected in each group on different days of the experiment (Table 1). The urine samples were subjected to ${ }^{1} \mathrm{H}$ NMR analysis to investigate the metabolic changes in urine caused by AA treatment. Representative $600 \mathrm{MHz}{ }^{1} \mathrm{H}$ NMR spectra from control and dosed groups are shown in Figure 2. The NMR spectra of mouse urine specimens showed different metabolic pattern after treatment for 10 days and 13 days in Experiment 1 and in Experiment 2, respectively. Multiparametric statistical analysis was applied to analyze ${ }^{1} \mathrm{H}-\mathrm{NMR}$ spectra and to investigate the differential metabolites between control and AA treated groups.

3.3. Multiparametric Statistical Analysis of ${ }^{1} H-N M R$ Data. A PLS-DA model was constructed to characterize the relationship among mouse groups. Figure 3 shows the first two components of the PLS-DA scores plots for both experiments. In Experiment 1, PLS-DA scores plots showed a good separation between the AA dosed groups (AA5, AA7.5) and control group (AA0) along the component 1 axis on day 10. We further used $R^{2}$ and $Q^{2}$ parameters to discriminate 


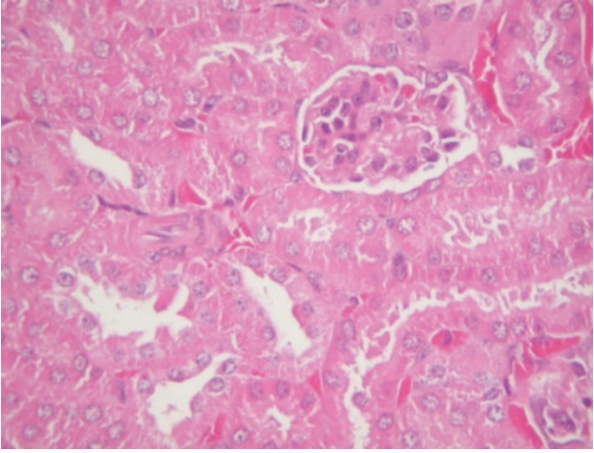

(a)

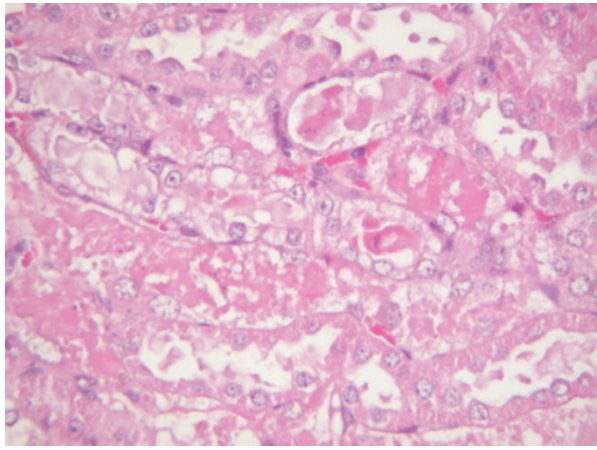

(c)

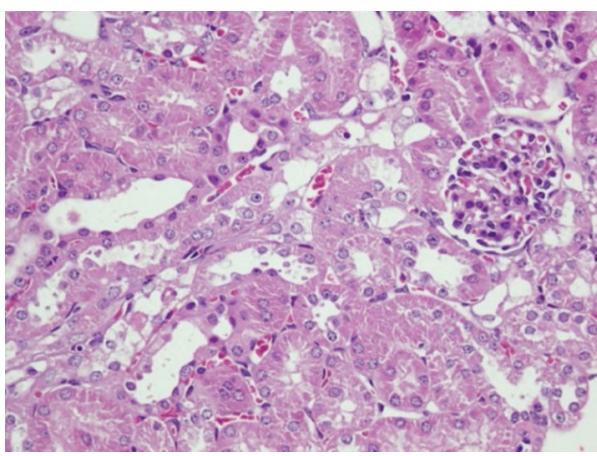

(e)

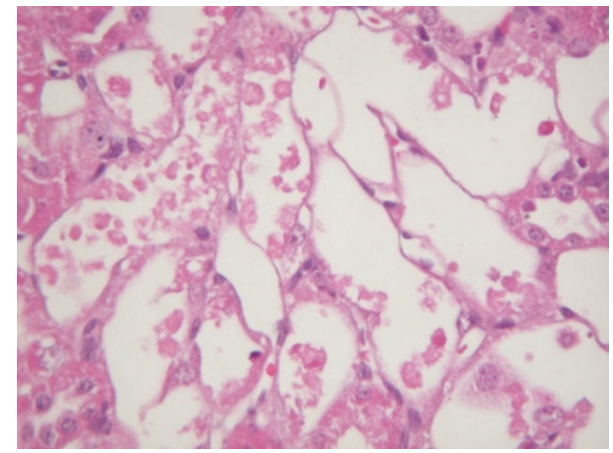

(b)

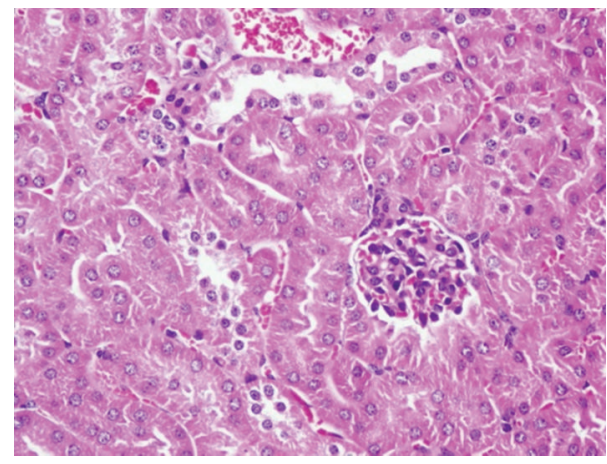

(d)

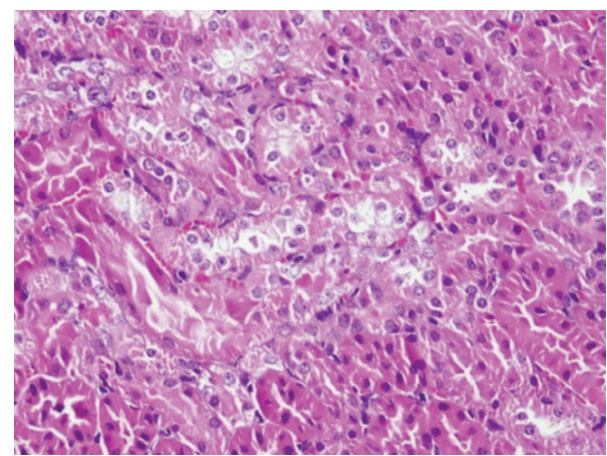

(f)

Figure 1: Representative histology (HE stain, magnification 400x) of kidney tissue from the different treatment groups. At 10 days in Experiment $1((\mathrm{a})-(\mathrm{c}))$, no significant alterations of renal tubules and glomeruli in control group (a). After treatment with medium dose of AA $(5 \mathrm{mg} / \mathrm{kg} /$ day), kidneys revealed moderate to severe acute proximal tubular necrosis (b). After treatment with high dose of AA (7.5 mg/kg/day), kidneys showed moderately to severe acute proximal tubular necrosis (c). At 20 days in Experiment 2 ((d)-(f)), no significant alterations of renal tubules and glomeruli in control group (d). After treatment with Madouling, the kidney showed focal, slight acute proximal tubular degeneration with cellular swelling (e). The change in BFAJT showed acute proximal tubular hydropic degeneration which is similar to mice treated with Madouling (f) (both with AA dosage equivalent to $0.5 \mathrm{mg} / \mathrm{kg} /$ day).

and predict the metabolic pattern difference between every two groups. In the scores plot, the $R^{2}$ value represents the percent variance we extracted from the spectral data and the $Q^{2}$ value represents the group predictability. Here, we showed the group discrimination of AA5 versus AA0, also for AA7.5 versus AA0 (the $R^{2}$, and $Q^{2}$ values between AA0 and AA5 are 0.88 and 0.50 ; the $R^{2}$ and $Q^{2}$ values between AA0 and AA7.5 are 0.94 and 0.71). But the discrimination between AA7.5 and AA5 is poor (the $R^{2}$, and $Q^{2}$ values between AA5 and AA7.5 are 0.85 and -1.03$)$ as the $Q^{2}$ value is negative. The findings of multivariate analysis with PLSDA show a compatible group difference as found in renal pathology (Figure 3(a)). In Experiment 2, a scores plot of PLS-DA showed clustering of the three groups ( $\mathrm{C} 0, \mathrm{M} 0.5$, and BF0.5) at day 13 (Figure 3(b)). The $R^{2}$ and $Q^{2}$ values between $\mathrm{C} 0$ and $\mathrm{M} 0.5$ are 0.80 and 0.23 , while the $R^{2}$ and $Q^{2}$ values between C0 and BF5 are 0.77 and 0.12 , showing underlying metabolic perturbation between dosed groups and control group. However, these separations between the two dosed groups M0.5 and BF0.5 are weak (a negative $Q^{2}$ value of -0.65 

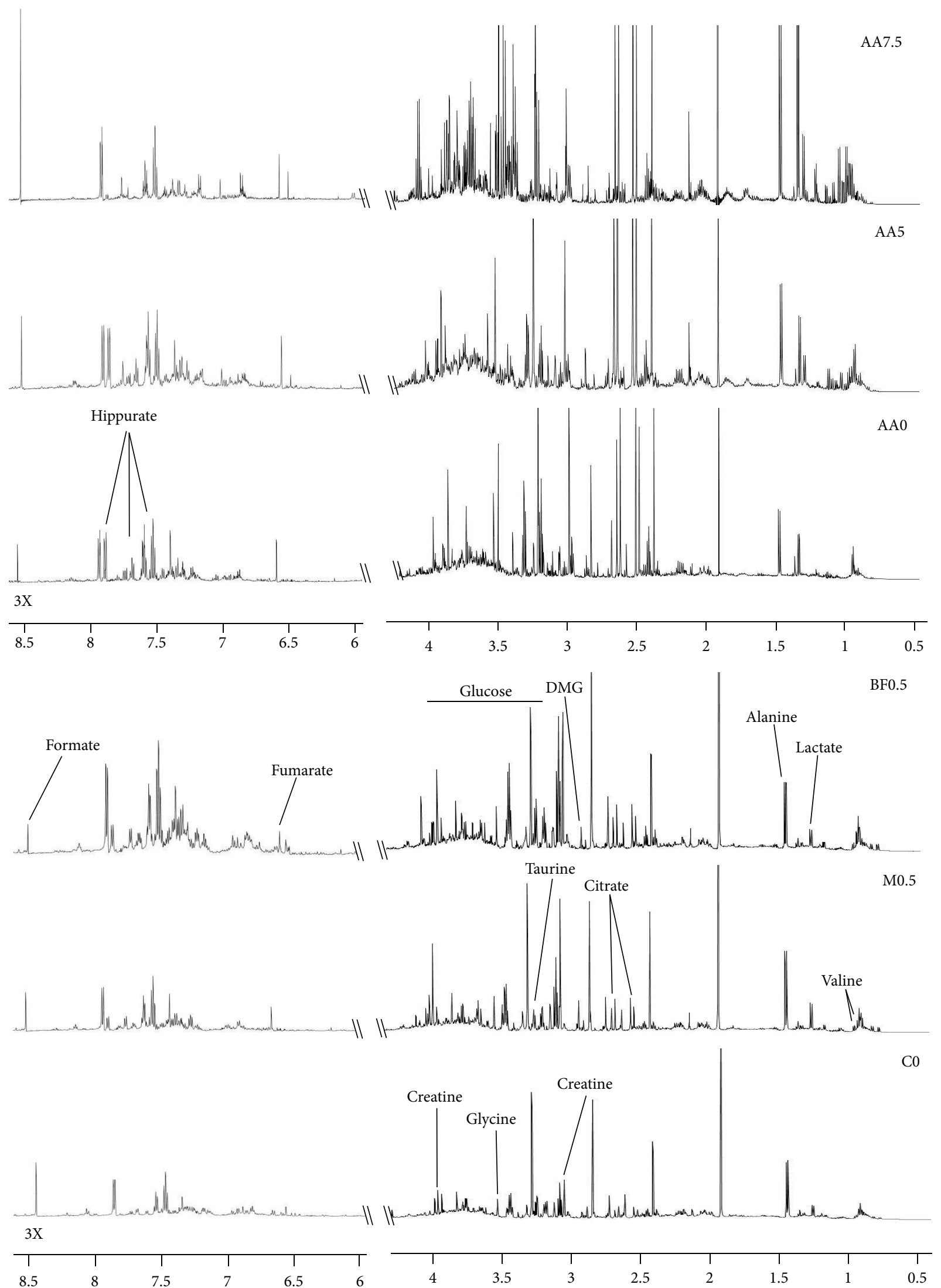

FIgURE 2: Representative ${ }^{1} \mathrm{H}$ NMR spectra of mouse urine after treatment for 13 days. Signals are assigned to their respective metabolites. The aromatic region $(\delta 6.0-8.5)$ was magnified three times in signal intensity as compared to the aliphatic region $(\delta 0.5-4.5)$. 


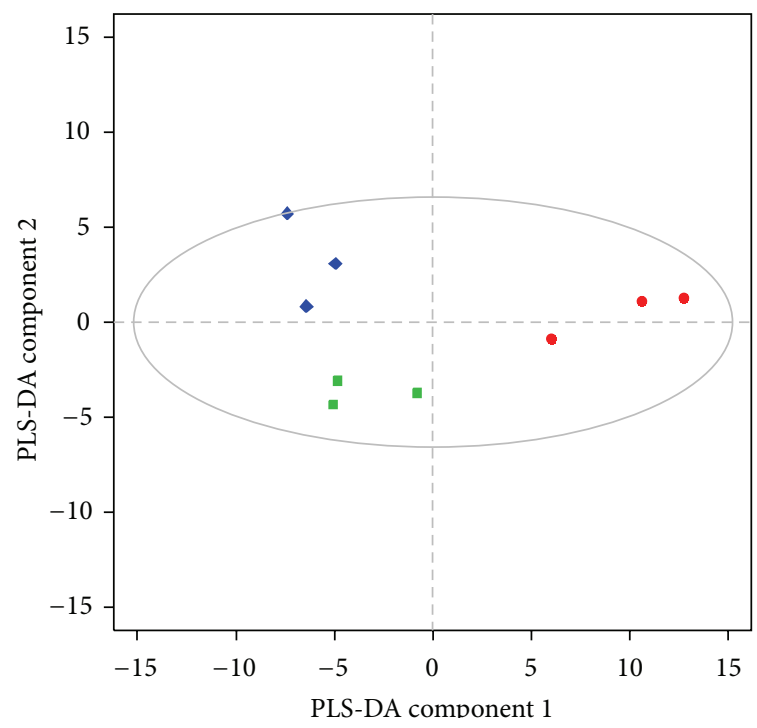

(a)

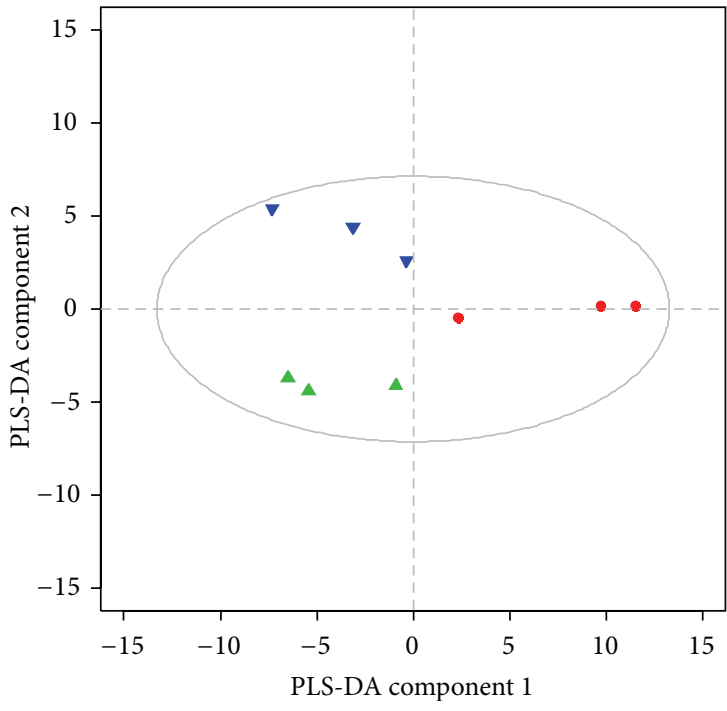

(b)

Figure 3: Partial least squares discriminant analysis (PLS-DA) scores plot showing clustering of different dosing groups using urinary ${ }^{1} \mathrm{H}$ nuclear magnetic resonance (NMR) dataset at day 10 in Experiment 1 (a) and at day 13 in Experiment 2 (b). Data symbols: AA0 • (red), AA5 (green), and AA7.5 (blue) of Experiment 1, C0 $\bullet$ (red), M0.5 $\boldsymbol{\Delta}$ (green), and BF0.5 $\boldsymbol{\nabla}$ (blue) of Experiment 2. The ellipse represents Hotelling's T2 with 95\% confidence.

and $R^{2}$ value of 0.97$)$. Compared to the pathological changes in Figure 1, we can discriminate between control group and dosed groups at even an earlier stage using PLS-DA prior to the pathological proof.

3.4. Metabolite Change in Influenced Pathway. Loadings plots were drawn to search for significant chemical shift variables. After identifying differential chemical signals, metabolites were assigned according to those chemical signals by Chenomx NMR Suite. Resonances with different intensity between the dosed and control groups were assigned to creatine, glycine, creatinine, TMAO (trimethylamine-Noxide), valine, hippurate, DMG (dimethylamine), citrate, lactate, alanine, glucose, fumarate, and formate (Figure 4). These metabolites and their relevant metabolic pathway were investigated for the underlying acute kidney injury from AA intoxication.

Detection of increased glucose and lactate in urine may suggest injuries in proximal renal tubules by nephrotoxicants. In the AA standard treated group, we observed an increase in glucose and lactate concentration in urine. In the Madouling and BFAJT treated groups, we found an increase in glucose concentration in urine, but the concentration of lactate did not show significant change. A number of nephrotoxicants have been studied by metabolomics [42-45]. In gentamicininduced nephrotoxicity in rats, there was increased in the concentration of glucose and lactate in urine $[42,43]$. As the lesion of gentamicin is mainly on the proximal renal tubule, characterized by a marked epithelial necrosis, the increase of glucose concentration in urine denotes perturbed proximal renal tubular reabsorption. Besides, the increased lactate concentration in urine indicates the loss of epithelial mitochondrial function. In a study of region specific nephrotoxins in rats, the increased concentration of glucose and lactate in urine was found in toxicants that injure the proximal renal tubule, such as hexachlorobutadiene, $\mathrm{HgCl}_{2}$, and sodium chromate, but this phenomenon was not found in nephrotoxicants that damage the region of renal papilla, such as propylene and 2-bromothanamine hydrobromide [44]. Since glucose and lactate concentration increased in AA treated groups in this study, it may suggest that the lesions caused by AA were on the proximal renal tubules. The metabolomic observation is correlated to the observation in the histopathological examination. In the M0.5 and BF0.5 groups, only glucose concentration was elevated in urine which may suggest a minor degree of proximal tubular lesion as we detected in the histopathological examination. In other metabolomic studies of AA nephrotoxicity, increase in glucose and lactate concentration in urine accompanying renal proximal tubular lesions has been detected in rats $[25,26]$. Another study indicated that the AA acute kidney injury caused diffuse degeneration of the proximal tubular epithelium [39].

Creatine was increased in the AA dosed groups. Increased creatine concentration in urine has been reported in subclinical renal papillary injury by 2-bromoethanamine hydrobromide [45]. The increase in creatine level in urine might be due to a variety of factors including creatine reabsorption, cell leakage, changes in both muscle mass, and bowel microflora metabolism [46]. The change in creatine concentration in this study may be related to renal papillary dysfunction with subclinical morphological change in the renal papillary region. 

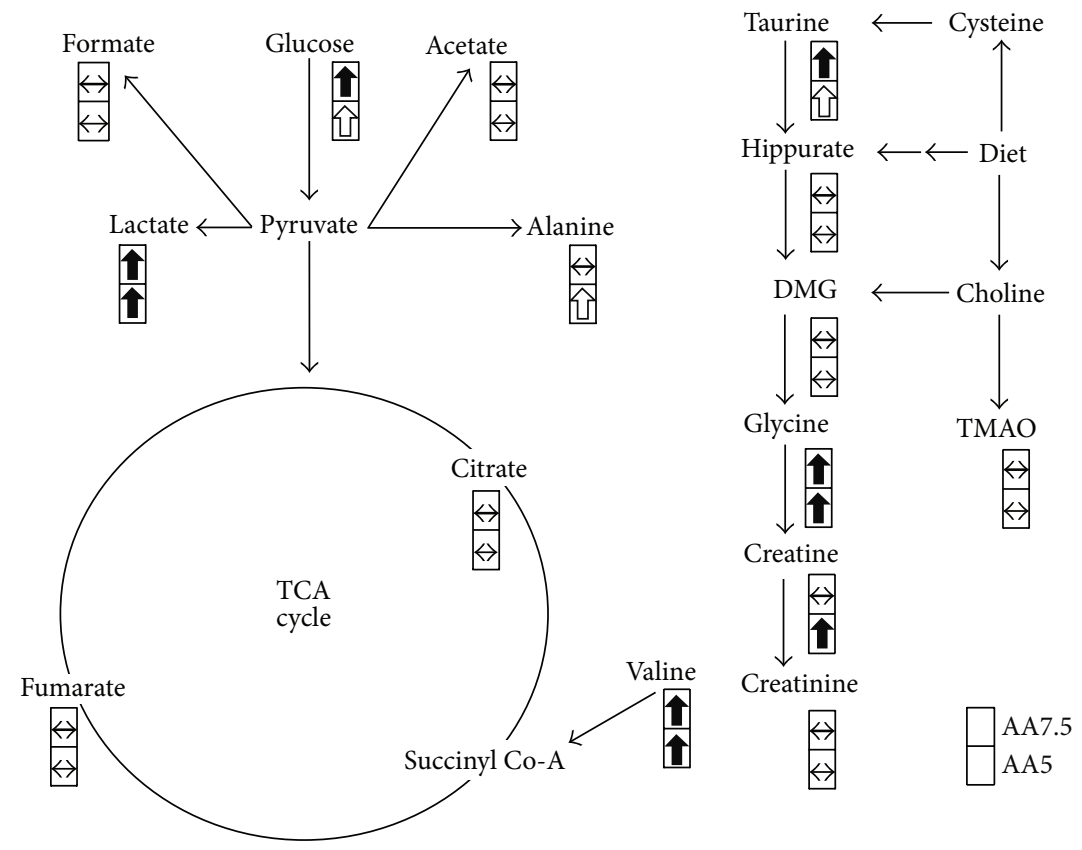

(a)
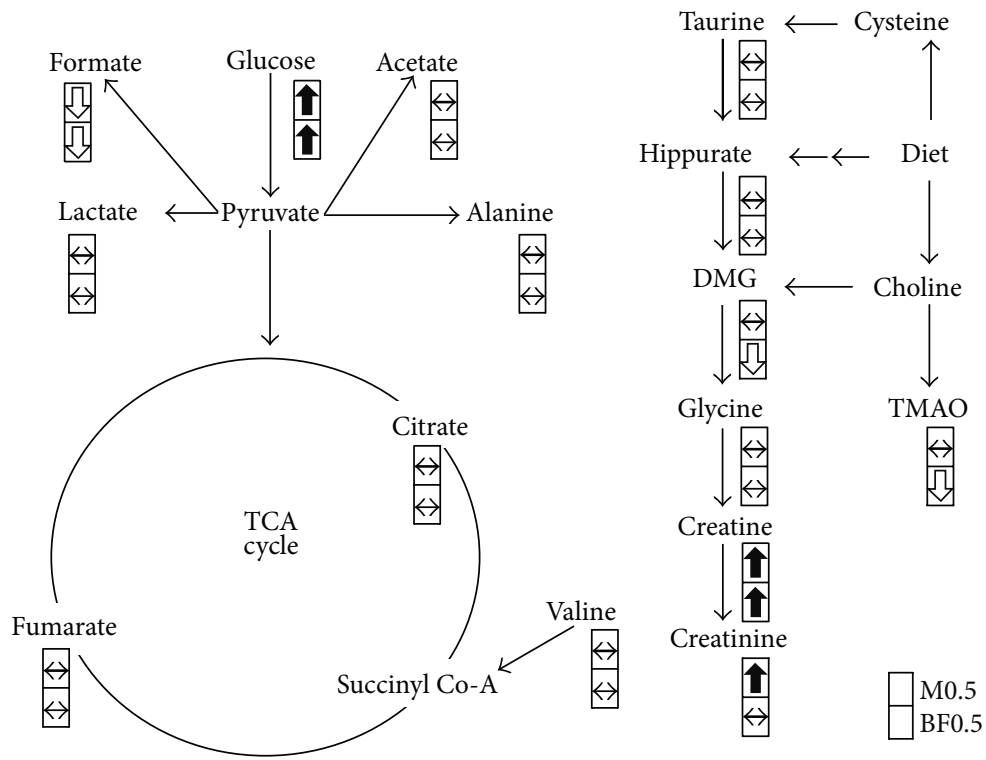

(b)

FIGURE 4: Perturbed metabolic pathways in response to AA substance exposure. (a) Energy metabolism, several amino acids, and creatinine are influenced. The metabolite concentrations are significantly increased for lactate, glucose, taurine, glycine, valine, and creatine for AA5 and AA7.5 on days 8-10 in Experiment 1. (b) Changes for M0.5 and BF0.5 on days 10-13 in Experiment 2. DMG dimethylglycine, TMAO trimethylamine- $\mathrm{N}$-oxide. Symbols in the cell represent relative concentration change of assigned metabolite between groups. They are significant increase (the black arrow up word)/decrease (the black arrow down word), $P<0.05$; nonsignificant increase (the white arrow up word)/decrease (the white arrow down word), fold change $>2$; no significant difference $(\leftrightarrow)$.

In herbal treated groups, both M0.5 and BF0.5 groups showed evidence of kidney injury from the changed concentration of glucose and creatine/creatinine. The similar trends with the AA standard group with lesser prominent changes of several metabolites (lactate, glycine and valine) may have resulted from the minor kidney injury.
In conclusion, AA standard, Madouling, and BFAJT were all nephrotoxicants as indicated by both metabolomics and pathological studies. The compositions of the compound remedy did not diminish the nephrotoxicity caused by AA. ${ }^{1} \mathrm{H}-\mathrm{NMR}$ was demonstrated as a convenient instrument to detect kidney injury, and it can be applied to evaluate the 
complicated metabolic response caused by herbal formulas. The control group and AA challenged groups can be classified by PLS-DA scoring plots of NMR spectra. The prediction strength from PLS-DA is stronger for the AA standard group as this group was administered higher amounts of AA. NMR metabolomics shows potential for early detection of AAN when coupled with multivariate pattern recognition analysis.

\section{Authors' Contribution}

C.-H. Kuo and Y. J. Tseng contributed equally to this paper.

\section{Acknowledgments}

This study was supported by a research Grant (CCMP96-RD044) from the Committee on Chinese Medicine and Pharmacy, Taiwan. Resources of the Laboratory of Computational Molecular Design and Detection, Department of Computer Science and Information Engineering, and Graduate Institute of Biomedical Engineering and Bioinformatics of National Taiwan University were used in performing this study.

\section{References}

[1] U. Mengs, "Acute toxicity of aristolochic acid in rodents," Archives of Toxicology, vol. 59, no. 5, pp. 328-331, 1987.

[2] F. D. Debelle, J. L. Vanherweghem, and J. L. Nortier, "Aristolochic acid nephropathy: a worldwide problem," Kidney International, vol. 74, no. 2, pp. 158-169, 2008.

[3] K. Hashimoto, M. Higuchi, B. Makino et al., "Quantitative analysis of aristolochic acids, toxic compounds, contained in some medicinal plants," Journal of Ethnopharmacology, vol. 64, no. 2, pp. 185-189, 1999.

[4] T. Cheung, C. Xue, K. Leung, K. Chan, and C. Li, "Aristolochic acids detected in some raw Chinese medicinal herbs and manufactured herbal products-a consequence of inappropriate nomenclature and imprecise labelling?" Clinical Toxicology, vol. 44, no. 4, pp. 371-378, 2006.

[5] V. M. Arlt, M. Stiborova, and H. H. Schmeiser, "Aristolochic acid as a probable human cancer hazard in herbal remedies: a review," Mutagenesis, vol. 17, no. 4, pp. 265-277, 2002.

[6] M. Heinrich, J. Chan, S. Wanke, C. Neinhuis, and M. S. J. Simmonds, "Local uses of Aristolochia species and content of nephrotoxic aristolochic acid 1 and 2-A global assessment based on bibliographic sources," Journal of Ethnopharmacology, vol. 125, no. 1, pp. 108-144, 2009.

[7] J. L. Vanherweghem, M. Depierreux, C. Tielemans et al., "Rapidly progressive interstitial renal fibrosis in young women: association with slimming regimen including Chinese herbs," The Lancet, vol. 341, no. 8842, pp. 387-391, 1993.

[8] J. L. Vanherweghem, F. Debelle, M. C. Muniz-Martinez, and J. Nortier, "Aristolochic acid nephropathy after Chinese herbal remedies," in Clinical Nephrotoxins, pp. 579-586, Kluwer Academic, New York, NY, USA, 2nd edition, 2004.

[9] J. L. Nortier, M. C. M. Martinez, H. H. Schmeiser et al., "Urothelial carcinoma associated with the use of a Chinese herb (Aristolochia fangchi)," The New England Journal of Medicine, vol. 342, no. 23, pp. 1686-1692, 2000.

[10] G. Gillerot, M. Jadoul, V. M. Arlt et al., "Aristolochic acid nephropathy in a Chinese patient: time to abandon the term
"Chinese herbs nephropathy"?" American Journal of Kidney Diseases, vol. 38, no. 5, p. E26, 2001.

[11] J. L. Nortier and J. L. Vanherweghem, "For patients taking herbal therapy-lessons from aristolochic acid nephropathy," Nephrology Dialysis Transplantation, vol. 22, no. 6, pp. 1512-1517, 2007.

[12] M. J. Martena, J. C. A. Van Der Wielen, L. F. J. Van De Laak, E. J. M. Konings, H. N. De Groot, and I. M. C. M. Rietjens, "Enforcement of the ban on aristolochic acids in Chinese traditional herbal preparations on the Dutch market," Analytical and Bioanalytical Chemistry, vol. 389, no. 1, pp. 263-275, 2007.

[13] X. J. Liu and S. B. Su, "Study of combination methods for formula composition of Chinese herbal medicines and their components," Journal of Chinese Integrative Medicine, vol. 7, no. 7, pp. 601-606, 2009 (Chinese).

[14] J. Qiu, "Traditional medicine: a culture in the balance," Nature, vol. 448, no. 7150, pp. 126-128, 2007.

[15] M. Liu, Y. Gao, R. Xiao, and B. L. Zhang, "Fuzzy mathematic quantitative law of composing principle in the study of traditional Chinese medicine," Yaoxue Xuebao, vol. 44, no. 1, pp. 3841, 2009 (Chinese).

[16] B. T. Schaneberg and I. A. Khan, "Analysis of products suspected of containing Aristolochia or Asarum species," Journal of Ethnopharmacology, vol. 94, no. 2-3, pp. 245-249, 2004.

[17] C. Laing, S. Hamour, M. Sheaff, R. Miller, and R. Woolfson, "Chinese herbal uropathy and nephropathy," The Lancet, vol. 368, no. 9532, p. 338, 2006.

[18] N. Zhang and M. Xie, "The nephrotoxicity in rats caused by Longdan Xiegan decoction," Zhongguo Zhongyao Zazhi, vol. 31, no. 10, pp. 836-839, 2006.

[19] C. Liu, A. Tseng, and S. Yang, Chinese Herbal Medicine: Modern Applications of Traditional Formulas, CRC Press, Boca Raton, Fla, USA, 2005.

[20] J. K. Nicholson, J. C. Lindon, and E. Holmes, “'Metabonomics': understanding the metabolic responses of living systems to pathophysiological stimuli via multivariate statistical analysis of biological NMR spectroscopic data," Xenobiotica, vol. 29, no. 11, pp. 1181-1189, 1999.

[21] O. Fiehn, "Metabolomics-the link between genotypes and phenotypes," Plant Molecular Biology, vol. 48, no. 1-2, pp. 155$171,2002$.

[22] G. A. N. Gowda, S. Zhang, H. Gu, V. Asiago, N. Shanaiah, and D. Raftery, "Metabolomics-based methods for early disease diagnostics," Expert Review of Molecular Diagnostics, vol. 8, no. 5, pp. 617-633, 2008.

[23] M. Mayr, "Metabolomics: ready for the prime time?" Circulation, vol. 1, no. 1, pp. 58-65, 2008.

[24] R. D. Beger, J. Sun, and L. K. Schnackenberg, "Metabolomics approaches for discovering biomarkers of drug-induced hepatotoxicity and nephrotoxicity," Toxicology and Applied Pharmacology, vol. 243, no. 2, pp. 154-166, 2010.

[25] X. Zhang, H. Wu, P. Liao, X. Li, J. Ni, and F. Pei, "NMR-based metabonomic study on the subacute toxicity of aristolochic acid in rats," Food and Chemical Toxicology, vol. 44, no. 7, pp. 10061014, 2006.

[26] Q. Liang, C. Ni, M. Xie et al., "Nephrotoxicity study of Aristolochia fangchi in rats by metabonomics," Journal of Chinese Integrative Medicine, vol. 7, no. 8, pp. 746-752, 2009 (Chinese).

[27] M. Chen, M. Su, L. Zhao et al., "Metabonomic study of aristolochic acid-induced nephrotoxicity in rats," Journal of Proteome Research, vol. 5, no. 4, pp. 995-1002, 2006. 
[28] M. Coen, E. Holmes, J. C. Lindon, and J. K. Nicholson, "NMR-based metabolic profiling and metabonomic approaches to problems in molecular toxicology," Chemical Research in Toxicology, vol. 21, no. 1, pp. 9-27, 2008.

[29] C. Shackelford, G. Long, J. Wolf, C. Okerberg, and R. Herbert, "Qualitative and quantitative analysis of nonneoplastic lesions in toxicology studies," Toxicologic Pathology, vol. 30, no. 1, pp. 93-96, 2002.

[30] R Development Core Team, R: A Language and Environment for Statistical Computing, R Development Core Team, Vienna, Austria, 2010.

[31] F. Dieterle, A. Ross, G. Schlotterbeck, and H. Senn, "Probabilistic quotient normalization as robust method to account for dilution of complex biological mixtures: application in ${ }^{1} \mathrm{H}$ NMR metabonomics," Analytical Chemistry, vol. 78, no. 13, pp. 42814290, 2006.

[32] U. G. Indahl, H. Martens, and T. Næs, "From dummy regression to prior probabilities in PLS-DA," Journal of Chemometrics, vol. 21, no. 12, pp. 529-536, 2007.

[33] B. H. Mevik and R. Wehrens, "The pls package: principal component and partial least squares regression in R," Journal of Statistical Software, vol. 18, no. 2, pp. 1-23, 2007.

[34] J. A. Westerhuis, E. J. J. van Velzen, H. C. J. Hoefsloot, and A. K. Smilde, "Discriminant Q2 (DQ2) for improved discrimination in PLSDA models," Metabolomics, vol. 4, no. 4, pp. 293-296, 2008.

[35] T. Lundstedt, E. Seifert, L. Abramo et al., "Experimental design and optimization," Chemometrics and Intelligent Laboratory Systems, vol. 42, no. 1-2, pp. 3-40, 1998.

[36] D. S. Wishart, D. Tzur, C. Knox et al., "HMDB: the human metabolome database," Nucleic Acids Research, vol. 35, no. 1, pp. D521-D526, 2007.

[37] M. E. Bollard, H. C. Keun, O. Beckonert et al., "Comparative metabonomics of differential hydrazine toxicity in the rat and mouse," Toxicology and Applied Pharmacology, vol. 204, no. 2, pp. 135-151, 2005.

[38] F. P. J. Martin, M. E. Dumas, Y. Wang et al., "A top-down systems biology view of microbiome-mammalian metabolic interactions in a mouse model," Molecular Systems Biology, vol. 3, p. 112, 2007.

[39] N. Sato, D. Takahashi, S. M. Chen et al., "Acute nephrotoxicity of aristolochic acids in mice," Journal of Pharmacy and Pharmacology, vol. 56, no. 2, pp. 221-229, 2004.

[40] X. Xue, Y. Xiao, H. Zhu et al., "Induction of P450 1A by 3-methylcholanthrene protects mice from aristolochic acid-Iinduced acute renal injury," Nephrology Dialysis Transplantation, vol. 23, no. 10, pp. 3074-3081, 2008.

[41] S. Shibutani, H. Dong, N. Suzuki, S. Ueda, F. Miller, and A. P. Grollman, "Selective toxicity of aristolochic acids I and II," Drug Metabolism and Disposition, vol. 35, no. 7, pp. 1217-1222, 2007.

[42] E. M. Lenz, J. Bright, R. Knight et al., "Metabonomics with 1H-NMR spectroscopy and liquid chromatography-mass spectrometry applied to the investigation of metabolic changes caused by gentamicin-induced nephrotoxicity in the rat," Biomarkers, vol. 10, no. 2-3, pp. 173-187, 2005.

[43] M. Sieber, D. Hoffmann, M. Adler et al., "Comparative analysis of novel noninvasive renal biomarkers and metabonomic changes in a rat model of gentamicin nephrotoxicity," Toxicological Sciences, vol. 109, no. 2, pp. 336-349, 2009.

[44] K. P. R. Gartland, F. W. Bonner, and J. K. Nicholson, "Investigations into the biochemical effects of region-specific nephrotoxins," Molecular Pharmacology, vol. 35, no. 2, pp. 242-250, 1989.
[45] E. Holmes, F. W. Bonner, and J. K. Nicholson, "Comparative studies on the nephrotoxicity of 2-bromoethanamine hydrobromide in the Fischer 344 rat and the multimammate desert mouse (Mastomys natalensis)," Archives of Toxicology, vol. 70, no. 2, pp. 89-95, 1995.

[46] A. A. Kandutsch and A. E. Russell, "Creatine and creatinine in tissues and urine of mice with hereditary muscular dystrophy," The American Journal of Physiology, vol. 194, no. 3, pp. 553-556, 1958. 


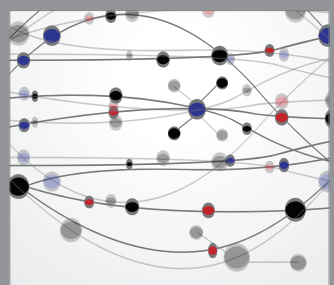

The Scientific World Journal
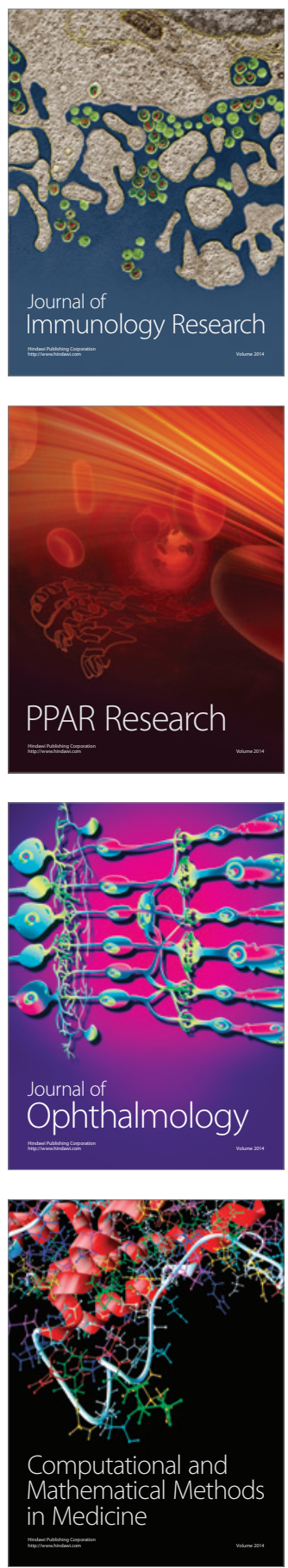

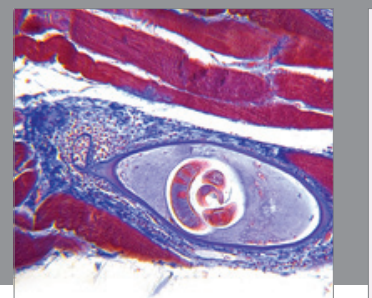

Gastroenterology

Research and Practice
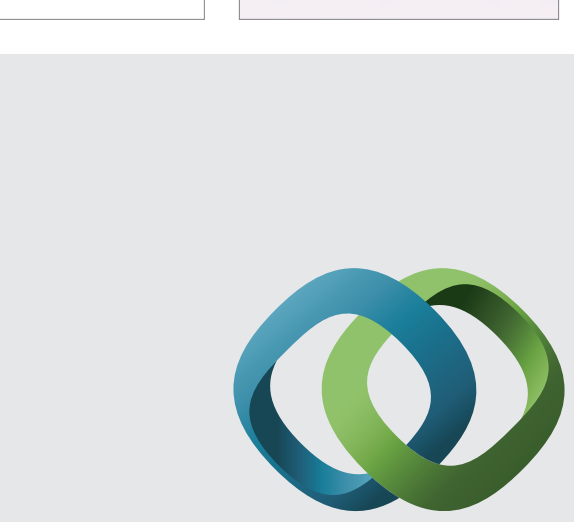

\section{Hindawi}

Submit your manuscripts at

http://www.hindawi.com
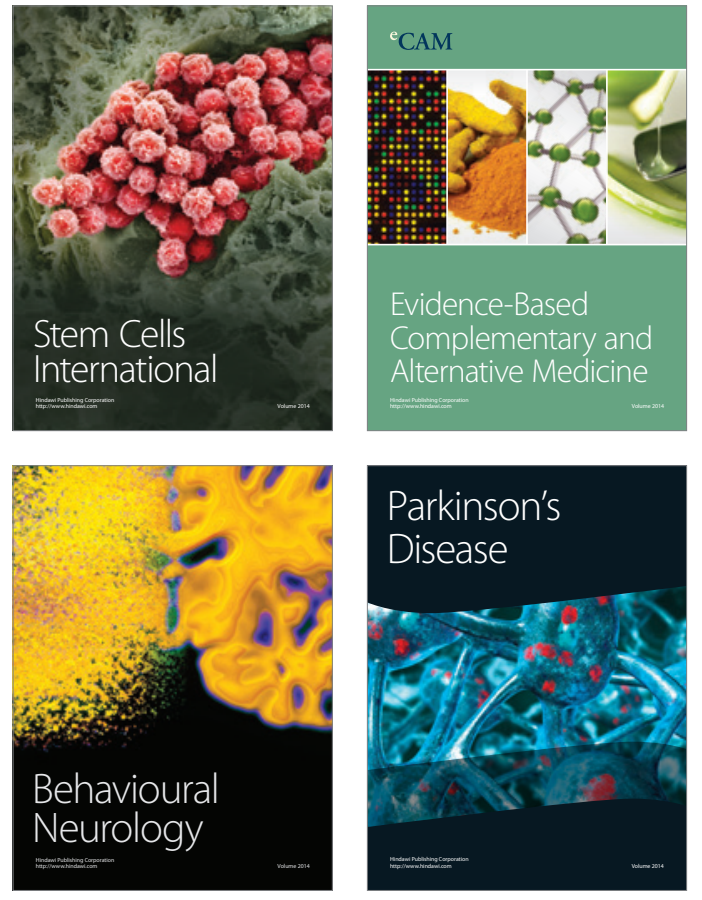
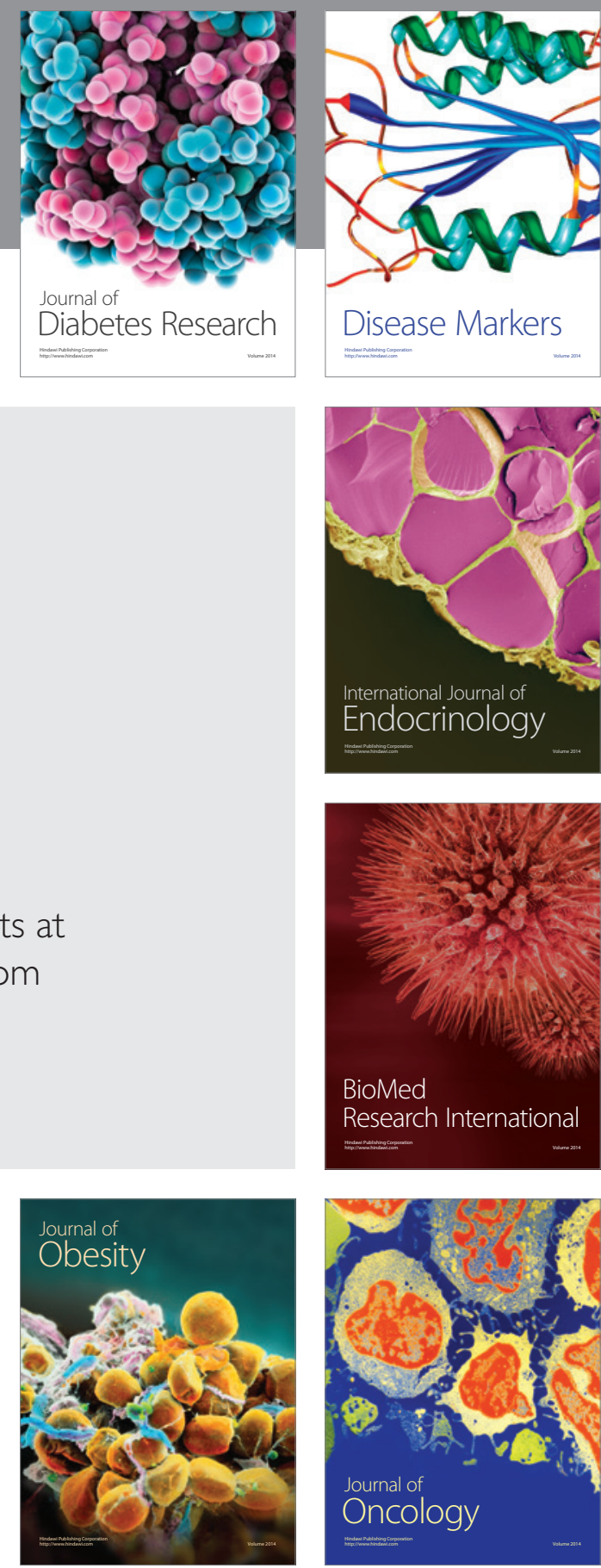

Disease Markers
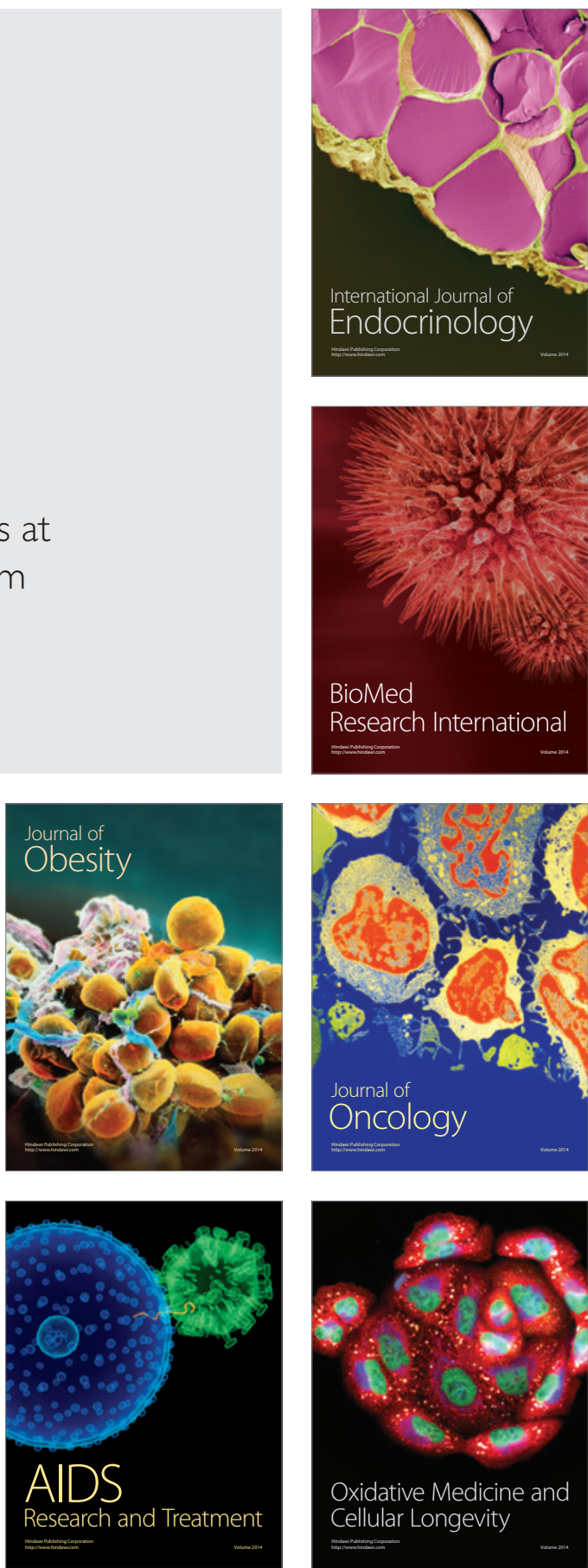